\title{
Reduced Universal Background Model for Speech Recognition and Identification System
}

\author{
Lachachi Nour-Eddine and Adla Abdelkader \\ Computer Science Department, Oran University, Algeria \\ \{Lach_Nour, AekAdla\} @Yahoo.fr
}

\begin{abstract}
Minimal Enclosing Ball (MEB) has a limitation for dealing with a large dataset in which computational load drastically increases as training data size becomes large. To handle this problem in huge dataset used for speaker recognition and identification system, we propose two algorithms using Fuzzy C-Mean clustering method. Our method uses divide-and-conquer strategy; trains each decomposed sub-problems to get support vectors and retrains with the support vectors to find a global data description of a whole target class. Our study is experimented on Universal Background Model (UBM) architectures in speech recognition and identification system to eliminate all noise features and reducing time training. For this, the training data, learned by Support Vector Machines (SVMs), is partitioned among several data sources. Computation of such SVMs can be efficiently achieved by finding a core-set for the image of the data.
\end{abstract}

Keywords: Quadratique Programming (QP), Support Vector Machines (SVMs), Minimum Enclosing Ball (MEB), core- set, kernel methods, Fuzzy CMean.

\section{Introduction}

Support vector machines (SVMs) [1], are a powerful technique for classification and regression. Training an SVM is usually posed as a quadratic programming (QP) problem to find a separation hyper-plane which implicates a matrix of density $n \times n$, where $n$ is the number of points in the dataset. This needs huge quantities of computational time and memory for large datasets, so the training complexity of SVM is highly dependent on the size of a dataset.

Here, we explore a technique that the training data, learned by Support Vector Machines (SVMs), is partitioned among several data sources. Computation of such SVMs can be efficiently achieved by finding a core-set for the image of the data in the feature space. However, in the standard technique that uses two classes SVM, a certain kernel functions and kernel methods are used to handle large datasets.

Many kernel methods can be equivalently formulated as minimal enclosing ball (MEB) problems in computational geometry by adopting an efficient approximate MEB algorithm. We obtain provably approximately optimal solutions with the idea of core-sets. 
The goal of this paper is to develop an alternative method based on a recently proposed equivalence between SVMs and Minimal Enclosing Ball (MEB) problems from which important improvements on training efficiency has been reported [2] [3] for large-scale datasets. We directly focus on multi-class problems exploring two methods to extend binary SVMs to the multi-category setting which preserve the equivalence between the model and MEBs. Algorithms to compute SVMs based on the MEB equivalence are based on the greedy computation of a core-set, a typically small subset of the data which provides the same MEB as the full dataset. Then, we formulate new multiclass SVM problem using core-sets for reduce large datasets which can be considered optimally matched to the input demands of different background architectures of speaker systems. The core idea of these two approaches is to adopt multiclass SVMs formulation and Minimal Enclosing Ball to reduce dataset without data noise.

This paper is presented as follows: section 2 introduces Minimal Enclosing Balls. Section 3 treats SVMs. Section 4 concerns Multiclass extension. Sections 5 presents the MEB and L2-SVMs equivalence in Multiclass approach. In section we discuss on the Fuzzy C-Mean clustering algorithm. The formulation of two algorithms that is the goal of our study is in section 7. Section 8 provides the experimental methodology and finally section 9 summarizes the conclusions.

\section{Minimal Enclosing Balls (MEB) and Core-Set}

Given a training dataset $S=\left\{\left(x_{i}, y_{i}\right)\right\}_{i=1}^{I}$ where $x_{i} \in \mathbb{R}^{d}$ and $y_{i} \in\{+1,-1\}$. To simplify the notation let us denote the pair $\left(x_{i}, y_{i}\right)$ as $\tilde{z}_{i}$. Now the training dataset can be denoted as $S=\left\{\tilde{z}_{i}\right\}_{i=1}^{I}$. Let $\tilde{Z}$ be a space equipped with a dot product $\tilde{z}_{i}^{T} \tilde{z}_{j}$ corresponding to norm $\|\tilde{z}\|^{2}=\tilde{z}^{T} \tilde{z}$. We define the ball $\mathcal{B}(c, R)$ of center $c \in \tilde{Z}$ and radius $R$ in $\mathbb{R}$ as the subset of points $\tilde{z} \in \tilde{Z}$ for which $\|\tilde{z}-c\|^{2} \leq R^{2}$. The minimalenclosing ball [3] of a set of points $S=\left\{\tilde{z}_{i}: i \in I\right\}$ in $\tilde{Z}$ is in turn the ball $\mathcal{B}^{*}\left(S, c^{*}, R^{*}\right)$ of smallest radius that contains $S$, that is, the solution to the following optimization problem.

$$
\begin{gathered}
\min _{R, c} R^{2} \\
s t:\|\tilde{z}-c\|^{2} \leq R^{2} \quad \forall \tilde{z} \in S
\end{gathered}
$$

After introducing Lagrange multipliers we obtain from the optimality conditions the following dual problem

$$
\begin{gathered}
\min _{\alpha} \sum_{i, j \in I} \alpha_{i} \alpha_{j} \tilde{z}_{i}^{T} \tilde{z}_{j}-\sum_{i \in I} \alpha_{i} \tilde{z}_{i}^{T} \tilde{z}_{i} \\
s t: \alpha_{i} \geq 0, \quad \sum_{i} \alpha_{i}=1 \quad \forall i \in I
\end{gathered}
$$

if we consider that $\sum_{i \in I} \alpha_{i} \tilde{z}_{i}^{T} \tilde{z}_{i}=\kappa$ a constant, we can drop it from the dual objective in (2), we obtain a simpler QP problem

$$
\begin{gathered}
\min _{\alpha} \sum_{i, j \in I} \alpha_{i} \alpha_{j} \tilde{z}_{i}^{T} \tilde{z}_{j} \\
\text { st: } \alpha_{i} \geq 0, \quad \sum_{i} \alpha_{i}=1 \quad \forall i \in I
\end{gathered}
$$

This is a QP problem. In [3], it shows that the primal variables $c$ and $R$ can be recovered from the optimal $\alpha$ as $c=\sum_{i=1}^{I} \alpha_{i} \tilde{z}_{i}, R=\sum_{i, j \in I} \alpha_{i} \alpha_{j} \tilde{z}_{i}^{T} \tilde{z}_{j}$ 


\subsection{Core-Set for MEB}

The algorithm of Bãdoiu and Clarkson [4] approximate the solution to this problem exploits the ideas of core-set and $\epsilon$-approximation to the minimal enclosing ball of a set of points. If we consider $S$ a set of $I$ points in $\mathbb{R}^{d}, R$ is the radius of $M E B(S)$, There exist a subset $C_{S} \subset S$ st:

- the size of $C_{S}$ is less $\frac{2}{\epsilon}$

- the center $c\left(C_{S}\right)$ of $M E B\left(C_{S}\right)$ satisfies $d\left(z, c\left(C_{S}\right)\right) \leq(1+\epsilon) R, \forall z \in S$

Such a subset $C_{S}$ is a core-set of $S$ for $M E B$. Then a core-set is a subset $C_{S}$ of $S$ such that:

- $\quad$ the size of $C_{S}$ does not depend on $I$ or $d$

- the solution for $C_{S}$ is an approximation of the solution for $S$.

$\epsilon$-coreset: The solution for $C_{S}$ is within $\epsilon$ of the solution for $S$.

Here we present the most usual version of the algorithm [4].

Algorithm 1. Bãdoiu-Clarson Algorithm

1: Initialize the core-set $C_{S, \epsilon}$.

2: Compute the minimal-enclosing-ball $\mathcal{B}\left(C_{S}, C, R\right)$ of the core-set $C_{S, \epsilon}$.

3: while $A$ point $\tilde{z} \in S$ out of the ball $\mathcal{B}(C, c,(1+\epsilon) R)$ exist do

4: Include $\tilde{z}$ in $C_{S, \epsilon}$.

5: Compute the minimal-enclosing-ball $\mathcal{B}\left(C_{S}, c, R\right)$ of the core-set $C_{S, \epsilon}$.

6: end while

\section{Support Vector Machines (SVMs)}

Support Vector Machines (SVMs) [1] address the problem of binary classification by building a hyperplane to represent the boundary between the two classes. This hyperplane $f(z)=\left(w^{T} z+b\right)$ is built in a feature space $Z=\phi(X)$ implicitly induced from $X$ by means of a kernel function $k$ which computes the dot products $z_{i}^{T} z_{j}=\phi\left(x_{i}\right)^{T} \phi\left(x_{j}\right)$ in $Z$ directly on $X$. The so called $L 2-S V M$ chooses the separating hyperplane $f(z)$ by solving the following quadratic program:

$$
\begin{gathered}
\min _{w, b, \rho, \xi} \frac{1}{2}\left(\|w\|^{2}+b^{2}+C \sum_{i} \xi_{i}^{2}\right)-\rho \\
s t: y_{i} f\left(z_{i}\right) \geq \rho-\xi_{i} \quad \forall i \in I
\end{gathered}
$$

After introducing Lagrange multipliers, it can be shown that the latter problem is equivalent to solve. 


$$
\begin{gathered}
\min _{\alpha} \sum_{i, j \in I} \alpha_{i} \alpha_{j} K_{i j} \\
\text { st: } 0 \leq \alpha_{i}, \sum_{i} \alpha_{i}=1
\end{gathered}
$$

where $K_{i j}=y_{i} y_{j} k\left(x_{i}, x_{j}\right)+y_{i} y_{j}+\frac{\delta_{i j}}{c}, \delta_{i j}$ is the Kronecker delta function and $k\left(x_{i}, x_{j}\right)$ implements the dot-product $z_{i}^{T} z_{j}$.

The main appeal of the L2 implementation is that it supports a convenient reduction to a minimal enclosing ball (MEB) problem when the kernel used in the SVM is normalized, that is $k(x, x)=\kappa \forall x \in X$, where $\kappa$ is a constant [3].

\section{$4 \quad$ Multi-class Extensions}

In a multi-class problem, examples $\left\{x_{i}\right\}$ belong to a set of $L$ categories $c=$ $\left\{c_{k} ; k \in L\right\}$ with $L \geq 2$ and hence the two "codes" +1 and -1 used to denote the two sides of a separating hyperplane are no longer enough to implement a decision function.

There are two types of extensions to build multiclass SVMs :

One Against One strategy $(\mathrm{OAO})$ that reduce the multi-class problem to a set of binary classification problems

One Against All strategy (OAA) that re-casts the binary objective function to a multi-category problem.

Up to our knowledge the only proposal of this nature directly addressing the multiclass extension of L2-SVMs is in [10]. This extension preserves the reduction to a minimal-enclosing-ball problem characteristic of the binary L2-SVM, which is the key requirement of our algorithms. The formulation associates each class $c_{k}, k \in L$ of the problem looks for a projector $W$ operating on the feature space $Z=\phi(X)$ which should allow to recover a correct code for a given input $z$.

Let the training dataset be $S=\left\{\left(x_{i}, y_{i}\right)\right\}_{i=1}^{I}$ where $x_{i} \in R^{d}$ and $y_{i} \in R^{L}$ for some integers $d ; L>0$. i.e. we have $I$ training points whose labels are vector valued. For a given training task having $L$ classes, these label vectors are chosen out of the definite set of vectors $\left\{y_{1}, y_{2}, \ldots, y_{I}\right\}$. Now, for inputs $z=\phi(x)$ we can define the primal for the learning problem as

$$
\begin{gathered}
\min _{\alpha} \frac{1}{2}\left(\|W\|^{2}+\|b\|^{2}+C \sum_{i} \xi_{i}^{2}\right)-\rho \\
\text { st: } y_{i}^{T}\left(W^{T} z+b\right) \geq \rho-\xi_{i}^{2} \geq 0 \quad \forall i \in I
\end{gathered}
$$

Several selections are possible for the norm $\|W\|^{2}$. A common choice is the so called Frobenius norm $\|W\|^{2}=\operatorname{trace}\left(W^{T} W\right)$. Hence, the dual of the optimization problem obtained after introducing Lagrange multipliers is

$$
\begin{gathered}
\min _{\alpha} \sum_{i, j \in I} \alpha_{i} \alpha_{j} K_{i j} \\
s t: 0 \leq \alpha_{i}, \sum_{i} \alpha_{i}=1
\end{gathered}
$$

where $K_{i j}=y_{i}^{T} y_{j} k\left(x_{i}, x_{j}\right)+y_{i}^{T} y_{j}+\frac{\delta_{i j}}{c}, \delta_{i j}$ is the Kronecker delta function and $k\left(x_{i}, x_{j}\right)$ implements the feature dot-products $z_{i}^{T} z_{j}$. 


\section{MEB and Multi-class L2-SVMs Equivalence}

Now, suppose we are computing the minimal-enclosing-ball in feature space $\tilde{Z}=$ $\phi(X)$ which has been induced from $X$ by a mapping function $\phi: X \rightarrow \tilde{Z}$ and suppose we can compute dot products in $\tilde{Z}$ directly from $X$ using a kernel function $\tilde{k}\left(x_{i}, x_{j}\right)=\phi\left(x_{i}\right)^{T} \phi\left(x_{j}\right)=\tilde{z}_{i}^{T} \tilde{z}_{j}$. Additionally suppose that the kernel is normalized, i.e., $\forall x \in X, \tilde{k}(x, x)=\kappa$ with $\kappa \in \mathbb{R}$ a constant.

As it has seen above, the optimization problem (1) is equivalent to solve the following quadratic program

$$
\begin{gathered}
\min _{\alpha} \sum_{i, j \in I} \alpha_{i} \alpha_{j} \widetilde{K}_{i j} \\
\text { st: } \alpha_{i} \geq 0, \quad \sum_{i \in I} \alpha_{i}=1 \quad \forall i \in I
\end{gathered}
$$

where $\widetilde{K}_{i j}=k\left(x_{i}, x_{j}\right)$. This problem coincides with the binary L2-SVM problem (5) and its multi-class implementation (7) if we set $\tilde{k}\left(x_{i}, x_{j}\right)=y_{i} y_{j} k\left(x_{i}, x_{j}\right)+y_{i} y_{j}+\frac{\delta_{i j}}{C}$ in the binary case, and $\tilde{k}\left(x_{i}, x_{j}\right)=y_{i}^{T} y_{j} k\left(x_{i}, x_{j}\right)+y_{i}^{T} y_{j}+\frac{\delta_{i j}}{C}$ in the multi-category case. The key requirement of the latter equivalence is the normalization constraint on $\tilde{k}$. Note however that the binary and the multi-category case, $\tilde{k}$ is constant when the kernel used by the SVMs $k$ is, i.e., $\tilde{k}(x, x)=\kappa$ a constant. This is a property satisfied by any kernel of the form $k\left(x_{i}, x_{j}\right)=\exp \left(-\left\|x_{i}-x_{j}\right\|^{2} / \sigma^{2}\right)$ for Gaussian Radial Basis Function kernel, and Exponential Radial Basis Function kernel formulated as $k\left(x_{i}, x_{j}\right)=\exp \left(-\gamma\left\|x_{i}-x_{j}\right\|^{2}\right)$, which is the most commonly used in practice.

\section{Fuzzy C-Mean Clustering Algorithm}

Fuzzy C-Mean (FCM) is an unsupervised clustering algorithm that has been applied to wide range of problems involving feature analysis, clustering and classifier design. This algorithm is examined to analyse different systems based on the distance between the various input data points. And it is based on clustering which allows one piece of data to belong to two or more clusters. It is based on minimization of the following objective function:

$$
\begin{gathered}
J_{m}=\sum_{i=1}^{N} \sum_{j=1}^{C} u_{i j}^{m}\left\|x_{i}-c_{j}\right\|^{2} \\
1 \leq m \leq \infty
\end{gathered}
$$

where $m$ is any real number greater than $1, u_{i j}$ is the degree of membership of $x_{i}$ in the cluster $j, x_{i}$ is the $i^{t h}$ of d-dimensional measured data, $c_{j}$ is the d-dimension center of the cluster, and $\|*\|$ is any norm expressing the similarity between any measured data and the center. Fuzzy partitioning is carried out through an iterative optimization of the objective function shown above, with the update of membership $u_{i j}$ and the cluster centers $c_{j}$ by: 


$$
\begin{gathered}
u_{i j}=\frac{1}{\sum_{k-1}^{c}\left(\frac{\left\|x_{i}-c_{j}\right\|}{\left\|x_{i}-c_{k}\right\|}\right)^{\frac{2}{m-1}}} \\
C_{j}=\frac{\sum_{i=1}^{N} u_{i j}^{m} \cdot x_{i}}{\sum_{i=1}^{N} u_{i j}^{m}}
\end{gathered}
$$

This iteration will stop when $\max _{i j}\left\{\left|u_{i j}^{(k+1)}-u_{i j}^{(k)}\right|\right\}<\xi$, where $\xi$ is a termination criterion between 0 and 1 , whereas $k$ is the iteration steps. This procedure converges to a local minimum or a saddle point of $J_{m}$. The algorithm is composed of the following steps:

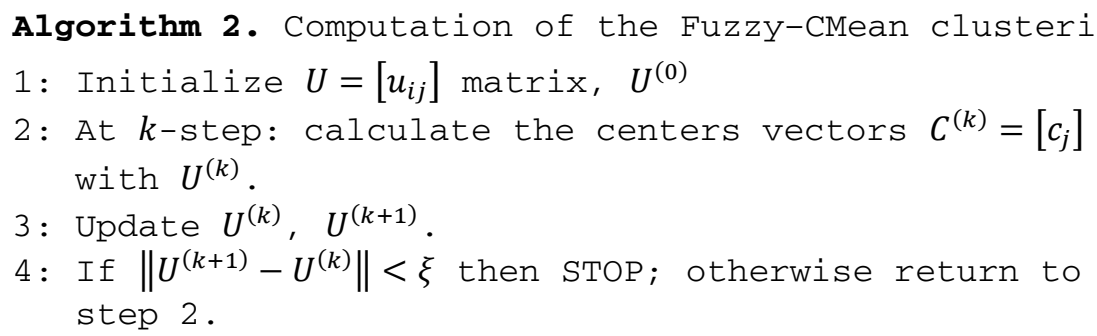

In this algorithm, data are bound to each cluster by means of a Membership Function, which represents the fuzzy behaviour of the algorithm [12], [13], [14]. To do that, the algorithm have to build an appropriate matrix named $U$ whose factors are numbers between 0 and 1 , and represent the degree of membership between data and centers of clusters.

\section{$7 \quad$ Reduced Data Approaches}

\subsection{Formulation}

The key idea of our method is to cast an SVM as a MEB problem in a feature space $\tilde{Z}=\phi(X)$ where the training examples are embedded via a mapping $\phi$. Hence, we first formulate an algorithm to compute the MEB of the images $\tilde{S}$ of $S$ in $\tilde{Z}$ when $S$ is decomposed in a collection of sub-sets $S_{j}$. Then we will instantiate the solution for classifiers supporting the reduction to MEB problems.

The algorithm is based on the idea of computing core-sets $\mathcal{C}_{j}$ for each set $\tilde{S}_{j}=$ $\phi\left(S_{j}\right)$ and taking its union $\mathcal{C}=\cup_{j} \mathcal{C}_{j}$ as an approximation to a core-set for $\tilde{S}=\cup_{j} S_{j}$. The generic procedure is depicted as algorithm (3). In a first step the algorithm extracts a core-set for each sub-set $S_{i}$. In the second step the MEB of the union of the core-sets is computed. 
Algorithm 3. Computation of the MEB of $\tilde{S}=\phi(S)$

Require: A partition of the set $S$ based Fuzzy-CMean clustering (algorithm 2) in a collection of subsets $S_{j}$

1: for Each subset $S_{j}, j=1, \ldots, p$ do

2: Compute a $\epsilon$-core-set $C_{j}$ for one of the two instantiation

3: end for

4: Join the core-sets $C=C_{1} \cup \ldots \cup C_{p}$

5: Compute the minimal enclosing ball of $C$. This is the Minimal enclosing ball of $\tilde{S}$ that define the reduced datasets.

For the computation of the core-sets we use the Bãdoiu and Clarkson (algorithm 1) described in the previous section.

\subsection{Instantiation for the OAO Multi-class Approach}

From the previous section we have that training a binary L2-SVM on a dataset $S$ is equivalent to build a minimal enclosing-ball of $S$ if $\phi(x)^{T} \phi(x)$ is implemented using the kernel $\tilde{k}\left(x_{i}, x_{j}\right)=y_{i} y_{j} k\left(x_{i}, x_{j}\right)+y_{i} y_{j}+\frac{\delta_{i j}}{C}$. The OAO procedure to obtain a multi-category SVM works by combining one binary SVM for each pair of classes. An instantiation of algorithm (3) would hence consist in computing core-sets for the subset of examples belonging to each pair of classes, and then joining them and finally recovering the binary model for this pair. However, since each class participates in $L$ models, core-sets for each pair of classes can be highly redundant overloading the data unnecessarily.

Algorithm 4. Computation of the MEB using OAO L2-SVMs

1: for Each subset $S_{n}, n=1, \ldots, p$ do

2: for Each Class $k=1, \ldots, L-1$ do

3: for Each Class $m=k+1, \ldots, L$ do

4: Let $S_{n}^{m k}$ the subset of $S_{i}$ corresponding to class $k$ and $m$.

5: Label $S_{n}^{m k}$ using the standard binary codes +1 and -1 for class $k$ and $m$ respectively

6: $\quad$ Compute a core-set $C_{n}^{m k}$ of $S_{n}^{m k}$ Using the kernel $\tilde{k}\left(x_{i}, x_{j}\right)=y_{i} y_{j} k\left(x_{i}, x_{j}\right)+y_{i} y_{j}+\frac{\delta_{i j}}{C}$

7: end for

8: end for

9: Take the union of the core-set inferred for each pair of classes $C_{n}=C_{n}^{m k} \cup \ldots \cup C_{n}^{m k}$ 
10: end for

11: Join core-set $C_{S}=C_{1} \cup \ldots \cup C_{p}$.

12: Compute the minimal enclosing ball of $C_{S}$ using the same kernel $\tilde{k}$

\subsection{Instantiation for the OAA Multi-class Approach}

The OAA implementation is defined by a single optimization which coincides with a MEB problem just by using the kernel $\tilde{k}\left(x_{i}, x_{j}\right)=y_{i}^{T} y_{j} k\left(x_{i}, x_{j}\right)+y_{i}^{T} y_{j}+\frac{\delta_{i j}}{C}$. The use of algorithm (3) is hence straight forward and consists in computing any dot product $\tilde{\phi}\left(x_{i}\right)^{T} \tilde{\phi}\left(x_{i}\right)=\tilde{k}\left(x_{i}, x_{i}\right)$ using this kernel. The instantiation is depicted as algorithm (5).

Algorithm 5. Computation of the MEB using OAA Multiclass L2-SVM

1: for Each subset $S_{n}, n=1, \ldots, p$ do

2: Label each example $x_{i} \in S_{n}$ with the code $y_{i k}$ assigned

to the class of $x_{i}$ and let $y_{i}$ such label

3: Compute a core-set $C_{n}$ of $S_{n}$ using the kernel $\tilde{k}\left(x_{i}, x_{j}\right)=y_{i}^{T} y_{j} k\left(x_{i}, x_{j}\right)+y_{i}^{T} y_{j}+\frac{\delta_{i j}}{C}$

4: end for

5: Join the core-sets $C_{S}=C_{1} \cup \ldots \cup C_{p}$.

6: Compute the minimal enclosing ball of $C_{S}$ using the same kernel $\tilde{k}$

\section{Experiments}

This section presents the performance of a speaker verification system based on the Gaussian Mixture described in [15]. We compare the performance of speaker verification system with three UBMs, the first one was created directly from the corpus in [16] (formerly known as Speaker Verification), and the two last later is the reduced of the original UBM from the application of our two algorithms developed in section 7. The kernel used for the two algorithms is the Gaussian Radial Basis Function with à fixed value of $\sigma$ with 0.50 . We have trained a 512-mixture gender-independent from each UBM with diagonal covariance matrices. Speaker GMMs are trained by adapting only the mean vectors from the UBM using a relevance factor $r$ of 16 .

Figure 3 shows the detection error trade-off (DET) curves for the three systems. The system based reduced GMM-UBM2 from One Against All multiclass L2-SVM outperforms the GMM-UBM with an equal-error-rate (EER) of $8.17 \%$, compared to $10.13 \%$ of the GMM-UBM2. The system based reduced GMM-UBM1 from OneAgainst-One L2-SVMs exhibits the best performance with an EER of $7.60 \%$. 


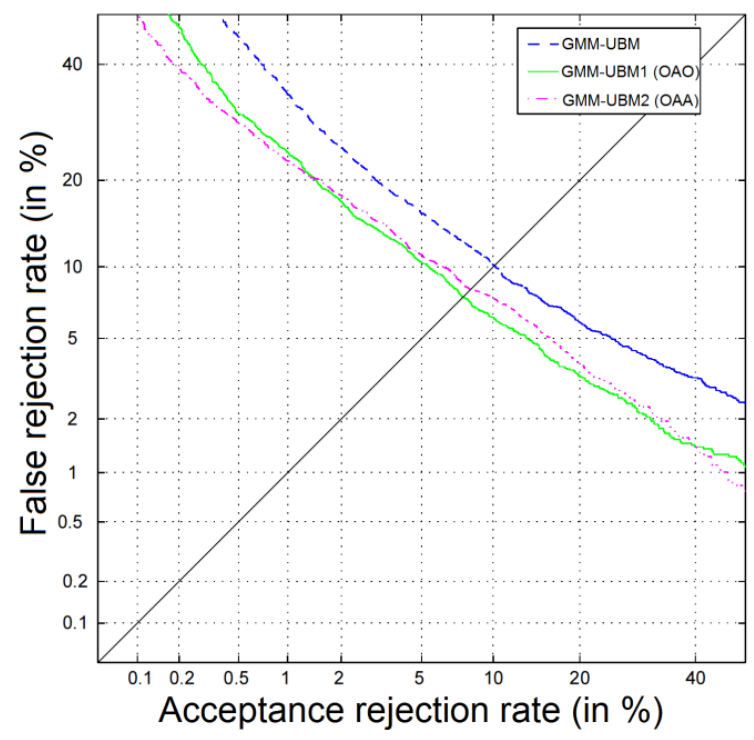

Fig. 1. DET curves for the speaker verification system using three UBMs

\section{Conclusion}

In this paper we have treated the Core-set with SVMs to reduce huge dataset. Thus, we have explained the characteristics of SVMs that make them valuable from the huge dataset point of view: first, SVMs are discriminative models, thus more appropriate for classification problems; second they have the advantage of being capable to deal with samples of a very higher dimensionality; and third, they exhibit an excellent generalization ability that makes them especially suitable to deal with noisy data.

Then, we proposed two algorithms that compute an approximation to the minimum enclosing ball of a given finite set of vectors. Both algorithms are especially wellsuited for large-scale instances of the minimum enclosing ball problem and can compute a small core-set whose size depends only on the approximation parameter.

We have explored two methods based on the computation of core-sets to train multi-category SVM models when the set of examples is fragmented. The main contribution has been to demonstrate through our experiments, that the methods proposed can reproduce the data with high accuracy where the noisy sample in huge dataset are eliminated, without complex and costly computation. SVMs based on core-sets have shown however important advantages in large-scale applications, which can hence be extended to distributed data-mining problems. A real contribution of this work has been a new direct implementation of multi-category SVMs supporting a reduction to a minimal-enclosing-ball (MEB) problem. Although the core-sets method exhibits always better prediction accuracy used with the OAO scheme, while the OAA implementation shows a lower complexity and it is better than the previous direct implementation proposed for MEB based SVMs. 


\section{References}

1. Schölkopf, B., Smola, A.J.: Learning with Kernels: Support Vector Machines, Regularization, Optimization, and Beyond. MIT Press, Cambridge (2001)

2. Kocsor, A., Kwork, J., Tsang, I.: Simpler core vector machines with enclosing balls. In: ICML 2007, pp. 911-918. ACM (2007)

3. Cheung, P.M., Kwok, J., Tsang, I.: Core vector machines: Fast SVM training on very large datasets. Journal of Machine Learning Research (6), 363-392 (2005)

4. Bãdoiu, M., Clarkson, K.L.: Optimal core-sets for balls. Computing Geometry Theory Application 1(40), 14-22 (2008)

5. Vapnik, V.: The nature of statistical learning theory. Springer (1995)

6. Hsu, C., Lin, C.: A comparison of methods for multiclass support vector machines. IEEE Transactions on Neural Networks 13(2), 415-425 (2002)

7. Crammer, K., Singer, Y.: On the algorithmic implementation of multiclass kernel-based vector machines. JMLR (2), 265-292 (2001)

8. Lee, Y., Li, Y., Wahba, G.: Multicategory support vector machines. Theory and application to the classification of microarray data and satellite radiance data. Journal of the American Statistical Association 99(465), 67-81 (2004)

9. Allende, H., Concha, C., Moraga, C., Nanculef, R.: Ad-svms: A light extension of SVMs for multicategory classification. International Journal of Hybrid Intelligent Systems 6(2), 69-79 (2009)

10. Asharaf, S., Murty, M., Shevade, S.K.: Multiclass core vector machine. In: ICML 2007, pp. 41-48. ACM (2007)

11. Shawe-Taylor, J., Szedmak, S.: Multiclass learning at one-class complexity. Technical Report, no 1508, School of Electronics and Computer Science, Southampton, UK (2005)

12. Al-Zoubi, M.B., Hudaib, A., Al-Shboul, B.: A fast fuzzy clustering algorithm. In: Proceedings of the 6th WSEAS Int. Conf. on Artificial Intelligence, Knowledge Engineering and Data Bases, Corfu Island, Greece, pp. 28-32 (2007)

13. Jain, A.K., Dubes, R.C.: Algorithms for Clustering Data. Prentice Hall Inc., Englewood Cliffs (1988)

14. Jain, A.K., Murty, M.N., Flynn, P.J.: Data Clustering - A review. ACM Computing Surveys 31(3) (September 1999)

15. Alkanhal, M., Alghamdi, M., Muzaffar, Z.: Speaker Verification based on Saudi Acceted Arabic Database. In: 9th International Symposium on Signal Processing and its Applications, ISSPA 2007, Sharjah, United Arab Emirate, pp. 1-4 (February 2007)

16. Speaker corpus in,

http://www.ll.mit.edu/mission/communication/ist/corpora/

speechCorpora.html 